\title{
Functional miniature human liver generated from stem cells
}

A functional human liver can be generated by transplanting small liver buds constructed from induced pluripotent stem cells (iPSCs) into mice, new research shows. The findings are a tentative step forward in the bid to produce laboratorygrown organs to address the critical shortage of donor organs available as treatment for end-stage organ failure.

Takanori Takebe and colleagues created in vitro liver buds (an early stage in liver organogenesis) by first differentiating human iPSCs into hepatic endoderm cells and then incubating these cells with a mixture of human endothelial and mesenchymal stem cells to recapitulate what naturally occurs during early organ development. The hepatic cells self-organized into a $3 \mathrm{D}$ liver bud that resembled those generated in vivo. "Our approach reconstitutes the cellular interactions that are important for natural liver progression, enabling the stem cells to efficiently differentiate into hepatocytes," explains Takebe.

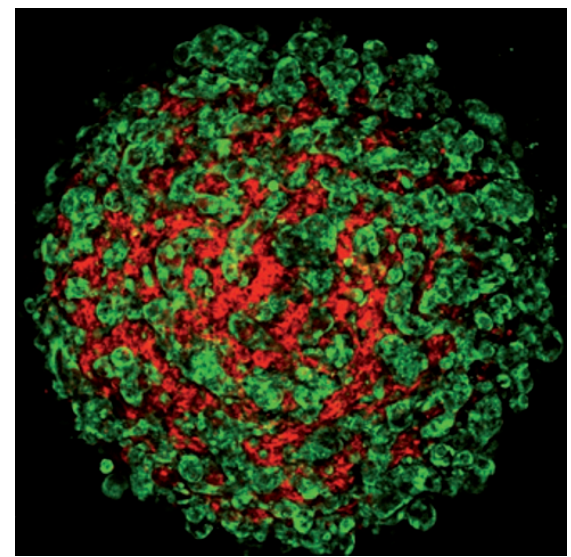

Human liver bud generated from human induced pluripotent stem cells. Courtesy of T. Takebe.

When transplanted into mice, the liver buds successfully engrafted; the vasculature within the bud connected quickly with the host blood vessels and was functional $48 \mathrm{~h}$ after transplantation. The liver buds then matured into tissue resembling an adult liver. Crucially, this tissue performed similar functions to a normal liver, including production of liver-specific metabolites (such as taurocholic acid) and metabolism of drugs (ketoprofen and debrisoquine). Moreover, mesenteric transplantation of liver buds improved survival of mice after liver failure induced by either diphtheria toxin or ganciclovir in two different mouse models.

Much work is needed before this approach can be applied in the clinic. "The bottleneck [in translation to clinical practice] is how to make huge amounts of liver buds to transplant back to patients, because the liver is one of the largest organs in our body and contains over 10-100 billion hepatocytes," says Takebe. The long-term function and safety of the liver buds will also need to be assessed.

\section{Katrina Ray}

Original article Takebe, T. et al. Vascularized and functional human liver from an iPSC-derived organ bud transplant. Nature doi:10.1038/nature12271 